Document downloaded from:

http://hdl.handle.net/10251/46182

This paper must be cited as:

Sánchez Martínez, F.; Orero Palomares, A.; Soriano Asensi, A.; Correcher Salvador, C.; González Martínez, AJ.; Hernández Hernández, L.; Moliner Martínez, L.... (2013). ALBIRA: A small animal PET/SPECT/CT imaging system. Medical Physics. 40(5):51906015190611. doi:10.1118/1.4800798.

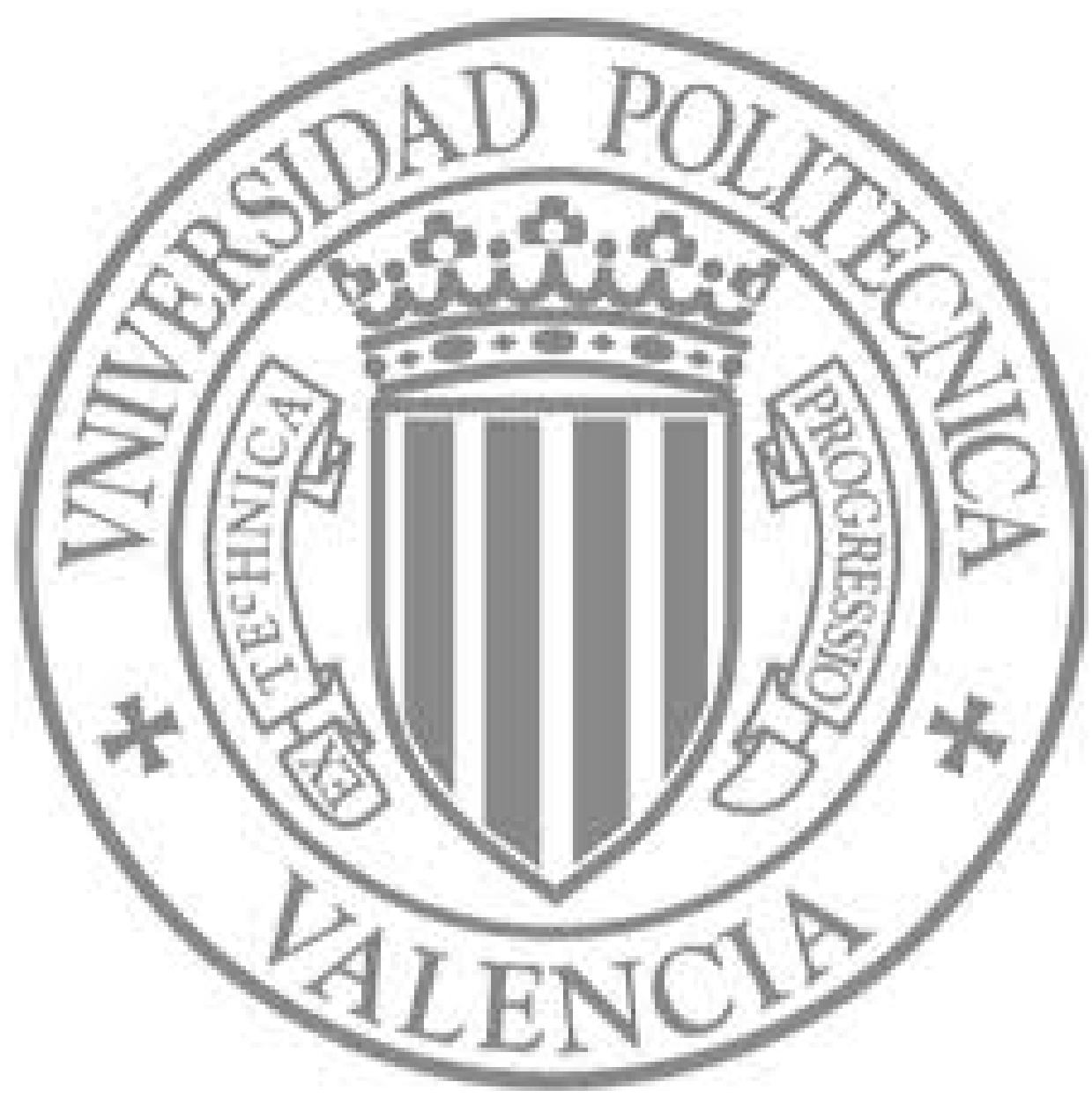

The final publication is available at

http://dx.doi.org/10.1118/1.4800798

Copyright American Association of Physicists in Medicine: Medical Physics 


\title{
ALBIRA: a small animal PET/SPECT/CT imaging system
}

\author{
F. Sánchez ${ }^{\text {a) }}$ A. Orero, A. Soriano \\ Instituto de Instrumentación para Imagen Molecular (I3M). Centro mixto CSIC - Universitat Politècnica \\ de València - CIEMAT, camino de Vera s/n, 46022 Valencia, Spain \\ C. Correcher \\ ONCOVISION, Centro de Investigación Príncipe Felipe. C/ Eduardo Primo Yúfera 3 Torre C. $3^{\text {a Planta, }}$ \\ E-46013 Valencia, Spain. \\ P. Conde, A. González, L. Hernández, L. Moliner, M.J. Rodríguez-Alvarez, L. F. Vidal, \\ J.M. Benlloch \\ Instituto de Instrumentación para Imagen Molecular (I3M). Centro mixto CSIC - Universitat Politècnica \\ de València - CIEMAT, camino de Vera s/n, 46022 Valencia, Spain
}

S. E. Chapman, W. M. Leevy

Notre Dame Integrated Imaging Facility, Notre Dame, IN 46556, USA

\begin{abstract}
:
Purpose: We have developed a trimodal PET/SPECT/CT scanner for small animal imaging. The gamma ray sub-systems are based on monolithic crystals coupled to multi-anode photomultiplier tubes (MA-PMTs), while CT comprises a commercially available micro-focus X-ray tube and a CsI scintillator 2D pixelated flat panel X-ray detector. In this study we will report on the design and performance evaluation of the multimodal system.
\end{abstract}

Methods: X-ray transmission measurements are performed based on cone-beam geometry. Individual projections were acquired by rotating the X-ray tube and the 2D flat panel detector, thus making possible a transaxial FOV of roughly $80 \mathrm{~mm}$ in diameter and an axial FOV of $65 \mathrm{~mm}$ for the CT system. The SPECT component has a dual head detector geometry mounted on a rotating gantry. The distance between the SPECT module detectors can be varied in order to optimize specific user requirements, including variable FOV. The PET system is made up of eight compact modules forming an octagon with an axial Field Of View (FOV) of $40 \mathrm{~mm}$ and a transaxial FOV of 80 $\mathrm{mm}$ in diameter.

The main CT image quality parameters (spatial resolution and uniformity) have been determined. In the case of the SPECT, the tomographic spatial resolution and system sensitivity have been evaluated with a ${ }^{99 \mathrm{~m}} \mathrm{Tc}$ solution using single-pinhole and multipinhole collimators. PET and SPECT images were reconstructed using threedimensional (3D) Maximum Likelihood and Ordered Subset Expectation Maximization (MLEM and OSEM)) algorithms developed by the authors, whereas the CT images were obtained using a 3D based FBP algorithm.

Results: CT spatial resolution was $85 \mu \mathrm{m}$ while a uniformity of $2.7 \%$ was obtained for a water filled phantom at $45 \mathrm{kV}$. The SPECT spatial resolution was better than $0.8 \mathrm{~mm}$ measured with a Derenzo-like phantom for a FOV of $20 \mathrm{~mm}$ using a 1-mm pinhole aperture collimator. The full width at half-maximum (FWHM) PET radial spatial resolution at the center of the field of view was $1.55 \mathrm{~mm}$. The SPECT system sensitivity for a FOV of $20 \mathrm{~mm}$ and $15 \%$ energy window was $700 \mathrm{cps} / \mathrm{MBq}\left(7.8 \times 10^{-2} \%\right)$ using a multi-pinhole equipped with 5 apertures $1 \mathrm{~mm}$ in diameter, whereas the PET absolute sensitivity was $2 \%$ for a $350-650 \mathrm{keV}$ energy window and a $5 \mathrm{~ns}$ timing window. Several animal images are also presented. 
Conclusions: The new small animal PET/SPECT/CT proposed here exhibits high performance, producing high-quality images suitable for studies with small animals. Monolithic design for PET and SPECT scintillator crystals reduces cost and complexity without significant performance degradation.

Keywords: nuclear medicine, radionuclide imaging, small animal imaging, integrated PET/SPECT/CT scanner

\section{I.INTRODUCTION}

A large number of small-animal Single Photon Emission Computed Tomography (SPECT) ${ }^{1-5}$ and Positron Emission Tomography (PET) ${ }^{6-15}$ systems have been developed and have become commercially available during the past years. Moreover, multimodality imaging has emerged as an important tool in Nuclear Medicine both for diagnostic and therapy follow up imaging. In this sense, efforts to develop multimodality preclinical imaging systems integrated in a common gantry have been undertaken. Currently developed devices are mainly based on SPECT/CT, PET/CT and PET/SPECT/CT multimodal systems. In this combination of modalities, Computed Tomography (CT) provides the anatomic context to assist in the interpretation of the functional PET or SPECT study. ${ }^{16} \mathrm{CT}$ morphological information can be used to obtain a finer spatial localization of the radiotracer distribution within the body. ${ }^{17}$

Small-animal models represent a critical bridge between discoveries at the molecular level and implementation of clinically relevant diagnostics or therapeutics. Although less attenuation and scatter in mice and rats than in humans can be expected in PET and SPECT scanners, the attenuation of detectable photons by soft tissue is estimated to be up to $50 \%$ when imaging ${ }^{125} \mathrm{I}$ and up to $25 \%$ when imaging ${ }^{99 \mathrm{~m}} \mathrm{Tc}$ in rat-sized objects. ${ }^{18}$ Furthermore, simulation studies suggest that scatter may contribute approximately $20 \%-25 \%$ to the total counts in a rodent-sized object for many isotopes used in SPECT studies. ${ }^{18,19}$ In this way, CT data can be used to obtain the attenuation coefficient map and scatter correction of the PET and SPECT images. In addition, when quantitative information on small target sites is needed, PET and SPECT images can suffer from an appreciable partial volume error that cannot be corrected without the knowledge of the target morphology. In this case, information from a morphological imaging technique, such as CT, allows for performing partial volume correction for tracer quantification. Examples of such preclinical trimodality systems are the Triumph ${ }^{\mathrm{TM}}$ Trimodality System, ${ }^{\mathrm{a}}$ VECTor/CT, ${ }^{\mathrm{b}}$ and Inveon PET/SPECT/CT. ${ }^{20}$

In this work we describe the ALBIRA small animal PET/SPECT/CT scanner, which was designed and developed at our laboratory. Its most innovative feature is the fact that the PET system is made up of monolithic scintillating crystals (non-pixelated). Most of the current PET devices are based on pixellated detectors, either on scintillation crystals or on CZT room temperature solid state detectors. Large continuous scintillation crystals were first successfully introduced in PET cameras by the University of Pennsylvania PET group ${ }^{21}$ but are not used in commercial small animal scanners.

\footnotetext{
${ }^{a}$ http://www.gammamedica.com/pre_triumph_trimodality_system.html

${ }^{\mathrm{b}}$ http://www.milabs.com/imaging-solutions/vector-simultaneous-spectpet/
} 
Since our group has prior experience in the use of continuous scintillating crystals in small gamma cameras ${ }^{22}$ we have extended the use of continuous scintillating crystals to our small animal PET and SPECT systems.

\section{SYSTEM DESIGN}

In the small animal PET/SPECT/CT that we present in this work, the subsystems are combined in a common gantry, with SPECT and CT placed at the rear and PET at the front (Fig. 1). The SPECT component is mounted perpendicularly to the CT component. The imaging bed has enough axial movement to image individually or with all three modalities in combination, thus allowing us to reach an axial FOV up to $170 \mathrm{~mm}$. The CT system consists of a commercially available micro-focus X-ray tube and a CsI(Tl) pixelated flat panel detector. The SPECT system utilizes a dual head detector geometry based on a gamma camera previously developed by our group. ${ }^{22}$ The PET subsystem has been developed by the authors and was fully tested and validated in a previous study. ${ }^{23}$

\section{II.A CT subsystem}

The X-ray CT subsystem uses a $50 \mathrm{kV}$ micro-focus X-ray source with a focal spot size of $35 \mu \mathrm{m}$ (Oxford Instruments XTF5011, X-Ray Technologies Inc., Scotts Valley, California, USA) and a $125 \mu \mathrm{m}$ beryllium exit window. The X-ray tube is sealed and has a fixed tungsten anode. The electron gun assembly is packaged inside a stainless steel lead-lined tube that provides X-ray shielding to $0.25 \mathrm{mR} / \mathrm{hr}$ at $5 \mathrm{~cm}$. Cooling oil is placed inside the tube to enhance heat dissipation. The micro-focus $\mathrm{x}$-ray source is operated in a continuous mode, delivering a $22^{\circ}$ cone $x$-ray beam with a maximum current of $1 \mathrm{~mA}$. An additional beam filtration with a $500 \mu \mathrm{m}$ thick aluminium plate was employed as well.

The CMOS flat-panel x-ray image sensor (Hamamatsu C7942) consists of a 2400x2400 pixelated array of $\mathrm{CsI}(\mathrm{Tl}) 200 \mu \mathrm{m}$ thick coupled to photodiode sensors, covering an active area of $120 \times 120 \mathrm{~mm}^{2}$, thus allowing a nominal resolution of $8 \mathrm{lp} / \mathrm{mm}$ (line pairs per millimetre). If no binning is performed the transfer rate is 2 frames per second increasing up to 9 frames per second when $4 \times 4$ binning is selected. A Phoenix D36 Frame-Grabber card (Active Silicon) is used to interface the sensor with the computer. Fixed distances from the X-ray tube to the isocenter $(290 \mathrm{~mm})$ and $\mathrm{x}$-ray detector $(425$ $\mathrm{mm}$ ) lead to a magnification factor of 1.46. This geometry allows a transaxial FOV of about $80 \mathrm{~mm}$ in diameter and an axial FOV of $65 \mathrm{~mm}$.

Four configuration modes are defined in the CT subsystem (Table I) that can be selected depending on the desired spatial resolution. The number of views determines the total scan time and the dose level. In normal daily operation, where voxel sizes ranging from $125 \mu^{3}$ to $500 \mu \mathrm{m}^{3}$ are considered, the optimal binning of the flat panel detector is $100 \times 100 \mu \mathrm{m}^{2}$. But, as long as higher resolutions are required and voxel sizes below 125 $\mu \mathrm{m}^{3}$ are used it is worth considering $50 \times 50 \mu \mathrm{m}^{2}$ detector binning.

Since the cone-beam image reconstruction algorithm is computationally intensive and a micro-CT with a flat-panel detector produces massive projection data, we have used a parallel data processing system to speed up the imaging reconstruction. The Feldkamp (FDK) algorithm ${ }^{24}$ was implemented to perform 3D images reconstruction in this study. 


\section{II.B SPECT subsystem}

The SPECT subsystem has a dual head detector geometry mounted on a rotating gantry where the CT is also placed. As was stated above, each detector head is based on a gamma camera previously developed by our group. The detector is made up of monolithic scintillating crystal. Scanners based on a pixelated design require the management of many small scintillating crystals, thus increasing cost and complexity. Moreover, there is a limitation on the smallest pixel size that can be obtained, thus limiting the intrinsic spatial resolution of the system. In addition, there is an unavoidable loss of sensitive area in these scanners due to the extra material needed to arrange the scintillating elements (pixels, blocks, and rings) which reduces the sensitivity of the system. Furthermore, in pixelated designs, energy resolution is a function not only of the intrinsic scintillation efficiency of the crystals, but also of the crystal size, with smaller cross-section crystals exhibiting greater light loss and, consequently, lower energy resolution. As the main drawback, continuous crystal design exhibits marked nonlinearities and non-uniformities that need to be treated by a proper calibration procedure.

Here, we will briefly describe the main design characteristics of the SPECT gamma detector (see Ref. 22 for further discussion on some details of the camera design). It is based on a multi anode photomultiplier tube (MA-PMT) H8500 from Hamamatsu Photonics, coupled with a continuous $\mathrm{CsI}(\mathrm{Na})$ with white-painted entrance face and black edges, 50x50x4 $\mathrm{mm}^{3}$ in size. The MA-PMT model has 64 anode pixels. The simultaneous digitization of the signals from all these anodes would considerably increase the electronic complexity of the system. Therefore, we reduce the number of signals to 4 by using a resistive current divider network, without compromising the obtained information. The centroid position $(X, Y)$ of the incident light pulse distribution on the photocathode is determined by the well-known Anger's equations. The sum of the four signals provides the total energy deposited by the incident gamma ray. However, the direct application of Anger's equations is not suitable for accurate position determination due to the nonlinearity of the system, especially when working with continuous crystals. For that reason, a position calibration of the SPECT head detector based on a 2D polynomial functional fit to the calibration data was performed. ${ }^{22}$ The crystal and the MA-PMT are surrounded by a lead layer $2 \mathrm{~mm}$ thick for gamma radiation shielding. Its intrinsic resolution is better than $1.5 \mathrm{~mm}$ and its energy resolution is about $13 \%$ at $140 \mathrm{keV}$.

Our SPECT subsystem can be operated with single- or multi-pinhole collimators.

Collimator geometry, including the distance from the source to the collimator, must be selected as a trade off between the spatial resolution and the sensitivity of the system.

Various collimator configurations were tested and, although sensitivity and spatial resolution for pinhole collimators significantly deteriorates as the radius of rotation increases, this also increases the FOV. The SPECT is designed in such a way that a linear actuator (Fig. 1) allows for a change in the source-head detector' distance in order

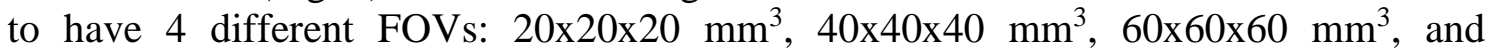
$80 \times 80 \times 80 \mathrm{~mm}^{3}$. We will refer to these as FOV20, FOV40, FOV60, and FOV80, respectively. These FOVs allow for magnification factors of 1.6 (FOV20), 1.0 (FOV40), and minification factors of 0.68 (FOV60) and 0.49 (FOV80). 
For the single-pinhole collimator a knife-edge type aperture $2 \mathrm{~mm}$ thick and made up of tungsten (Fig. 2a) has been used. It has an inner diameter of $1 \mathrm{~mm}$ and $2 \mathrm{~mm}$ for the outer diameter, allowing an acceptance angle of about $53^{\circ}$, with a focal length $f=31 \mathrm{~mm}$. In order to increase sensitivity without great loss of spatial resolution, the SPECT subsystem can be operated in a multi-pinhole configuration (Fig. 2b). Multi-pinhole, with a focal length $f=31 \mathrm{~mm}$, consists of 5 apertures $1 \mathrm{~mm}$ in diameter which were drilled on a block of $2 \mathrm{~mm}$ thick tungsten forming an $\mathrm{X}$. The external apertures are located at $4 \mathrm{~mm}$ from the central one. Pinhole collimators, both single- and multipinhole, were designed in such a way that they are easily interchangeable.

The events in the crystal are binned into a set of $0.5 \times 0.5 \mathrm{~mm}^{2}$ bins according to their impact position. The images were reconstructed using a 3D- OSEM algorithm developed by the authors.

\section{II.C PET subsystem}

As stated above, the PET component of our system is a scanner based on monolithic LYSO crystals that has been developed by the authors, whose design and performances has been extensively described in a previous work. ${ }^{23}$

It is made up of eight removable and identical modules forming an octagon. Each module contains a $10 \mathrm{~mm}$ thick monolithic LYSO crystal coupled to a MA-PMT H8500 from Hamamatsu Photonics. As we have stated above ( $\$$ II.B) we selected continuous crystals because these detectors can have higher efficiency than those based on pixelated crystals, together with an excellent spatial resolution. As we did for the SPECT subsystem, we reduce the number of MA-PMT signals, although in this case we extract 5 signals (4 devoted to position determination). An additional adder circuit, incorporated in the resistive anode network of the MA-PMT, measures the width of the light distribution which is directly related with the depth of interaction (DOI). ${ }^{23}$ This fifth anode signal is digitized to obtain the depth of interaction with a resolution (FWHM) of about $4 \mathrm{~mm}$. DOI information improves the spatial resolution (both radial and tangential) about 5\% (3\% for the axial direction) at the center of both axial and transaxial FOV and $13 \%$ (3\% for the axial direction) at the transaxial FOV edge.

The timing window can be varied from 3 to $11 \mathrm{~ns}$, although in normal operation mode this value is set to $5 \mathrm{~ns}$. PET subsystem has an axial FOV of $40 \mathrm{~mm}$ and a transaxial FOV of $80 \mathrm{~mm}$ in diameter and operates in 3D data acquisition mode. PET images can be reconstructed using 3D Maximum Likelihood and Ordered Subset Expectation Maximization (MLEM and OSEM) algorithms developed by the authors.

\section{II.D PET/SPECT/CT Integration}

In Fig. 3 we show the whole PET/SPECT/CT system in the common gantry after integration. The SPECT component was mounted perpendicular to the CT. Individual projections of SPECT and CT were acquired by rotating the common gantry using a computed controlled rotation system, with an angular precision of $0.01^{\circ}$.

The system is equipped with four interchangeable polystyrene $2.5 \mathrm{~mm}$ thick beds: one for rats, another for mice and a third for imaging mice heads. Finally, the fourth bed was especially developed for phantoms used for the system calibration. The photon attenuation due to bed material is about $6 \%$ in CT, $4 \%$ in SPECT and $2.5 \%$ in PET. A bed actuator allows the beds to go through the various components of the system (PETSPECT-CT). 


\section{METHOD}

\section{III.A SYSTEM CALIBRATION}

Prior to acquiring useful data with the PET/SPECT/CT system, the various subsystems were calibrated in position, energy and flood correction (normalization). These processes are referenced in the literature, and the authors have extensively described these procedures previously. ${ }^{22,23}$ For that reason, concerning system calibration we will only deal in this paper with the geometric calibration and mechanical alignment verification of the CT and SPECT subsystems, as the PET subsystem is fixed and is not allowed to have any rotation/translation movement.

\section{III.A.1 CT subsystem geometric calibration}

The procedure followed for the geometric calibration of the CT subsystem is based on the one described for SPECT geometric calibration by Beque et al. ${ }^{25,26}$. This procedure is based on the minimization of the squared differences between measured and estimated positions of a group of point like sources suitably placed in the FOV. During the minimization process a set of 13 parameters is adjusted, 7 of them (focal, focusisocenter, mechanical offset, $\mathrm{x}$ and $\mathrm{y}$ electrical shifts, tilt and twist) model the tomograph and the 6 remaining correspond to the placement and orientation of the point-like sources in the FOV. The model used to describe the CT tomograph is the same detailed in Ref. 25 , although instead of the ${ }^{99 \mathrm{~m}} \mathrm{Tc}$ point-like sources proposed in Ref. 25, we have used 18 stainless steel ball bearings with a diameter of $2 \mathrm{~mm}$. Moreover, a linear alignment of the ball bearings was preferred instead of the triangular alignment proposed in Ref. 25. Thus, only 5 parameters ( 3 position +2 angles) are required to locate the phantom in the FOV. The geometric parameters were obtained using a non linear least squares fit that minimizes the squared sum of the differences between measured and estimated positions of the ball bearings in the phantom.

\section{III.A.2 SPECT subsystem geometric calibration}

The SPECT geometric calibration procedure is based on the same method we have followed for CT subsystem ${ }^{25,26}$ conveniently adapted for dual head based on SPECT devices. ${ }^{27}$ The procedure is based on the measurements made with three point-like sources placed in such a way that their relative distances are accurately known as described in Refs. 25 and 26. The geometric parameters defining the SPECT subsystem were obtained in the same way as previously for CT (non linear least squares fit). On average, the deviations between real and measured distances were of the order of 0.25 $\mathrm{mm}$.

\section{III.B CT subsystem performance}

CT tomographic spatial resolution and uniformity were determined in order to characterize the CT subsystem. The frame time of the flat-panel detector to acquire a view of projection data has been kept constant at about $250 \mathrm{~ms}$ for all of the CT scans.

\section{III.B.1 Spatial resolution}

We have evaluated the spatial resolution of the CT subsystem using a thin gold wire 50 $\mu \mathrm{m}$ in diameter placed at the center of the FOV. Data acquisitions consisted of 1000 views over $2 \pi$ at a voltage of $45 \mathrm{kV}$. For this measurement voxel size of the reconstructed image was fixed at $8 \times 8 \times 8 \mu \mathrm{m}^{3}$. 


\section{III.B.2 Image uniformity and noise characteristics}

Image uniformity in a micro-CT depends on detector uniformity and the beam hardening effect. Although detector non-uniformity can be corrected by using the prescan data obtained without any subjects between the X-ray source and the detector, the detector noise and the time-dependent nature of the detector sensitivity and/or X-ray beam could cause non-uniformities in the reconstructed image. To evaluate image uniformity, we have used a polyMethylMethacrylate (plexiglass) cylindrical phantom filled with water. The fillable volume was $25 \mathrm{~mm}$ in length and $30 \mathrm{~mm}$ in diameter. After reconstructing the image, we evaluated the uniformity response by:

$$
\operatorname{DEV}(\%)=100 \cdot \frac{C T_{95}-C T_{5}}{C T_{50}}
$$

Absolute deviation $D E V$ reflects the variation range in a volume of interest (VoI). In order to avoid the undesirable effect of outlying values, the 95th $\left(\mathrm{CT}_{95}\right), 50$ th $\left(\mathrm{CT}_{50}\right)$ and 5th $\left(\mathrm{CT}_{5}\right)$ percentiles of the CT numbers for the voxels within the VoI have been considered in (1). Thus the $\mathrm{CT}_{95}$ is the CT number value below which the $95 \%$ of the voxel attenuation values (measured as CT numbers) in the VoI fall.

The image noise was evaluated by:

$$
N(\%)=100 \cdot \frac{\sigma}{\overline{C T}},
$$

where $\sigma$ and $\overline{C T}$ are the standard deviation and the mean value of the CT number, respectively, for the voxels within the selected VoI. The selected VoI was $20 \mathrm{~mm}$ in diameter, and $20 \mathrm{~mm}$ in length while the voltage was set to $45 \mathrm{kV}$.

\section{III.C SPECT subsystem performance}

In order to fully characterize the proposed SPECT design based on continuous CsI(Na) scintillation crystals, its sensitivity and tomographic spatial resolution across the FOV were determined. In all measurements, projection data were acquired in the step-andshoot mode with 60 views (30 views for each individual camera head). Time acquisition can be selected by the user, typically from 30 up to 90 seconds per view. This implies a total acquisition time typically between 15 up to 45 minutes. The dual-energy window method $^{28}$ was used for scatter correction of the data $(95-110 \mathrm{keV}$ window for scatter component determination was used, with slight variations if required by the data energy spectrum). The scatter correction method was tested by using a glass capillary tube with an outer/inner diameter of $3 / 1 \mathrm{~mm}$ and $70 \mathrm{~mm}$ in length. The glass capillary tube was placed $4 \mathrm{~mm}$ off-axis inside two different phantoms (cylinders made of plexiglass and teflon $30 \mathrm{~mm}$ in diameter and $70 \mathrm{~mm}$ in length). Scatter correction for SPECT recorded data allowed for an improvement in spatial resolution of about $3.5 \%$.

\section{III.C.1 Sensitivity}

A specific phantom for the measurement of the SPECT sensitivity was designed and built. It uses a cylinder $4 \mathrm{~mm}$ in length and $4 \mathrm{~mm}$ in diameter as fillable volume. Due to their reduced size, scatter and attenuation effects can be considered negligible. Total acquisition time was $15 \mathrm{~min}$ using a solution of ${ }^{99 \mathrm{~m}} \mathrm{Tc}$ as radioactive source. The initial 
activity was 13.6 and $12.7 \mathrm{MBq}$ for the single- and multi-pinhole configurations, respectively. The activity source was accurately determined in a dose calibrator (ISOMED 2010 by MED Nuklear-Medizinetechnik Dresden GmbH). We have studied sensitivity variation with the energy window for the FOV20 configuration in order to determine the optimal energy window. Once the optimal window energy was determined, the SPECT sensitivity was measured at the various FOVs considered for both single- and multi-pinhole configurations.

\section{III.C.2 Spatial resolution}

We have designed and built three Derenzo-like phantoms made of plexiglass to determine spatial resolution capabilities for the different FOVs considered (§II.B). The first one is a $20 \times 20 \times 5 \mathrm{~mm}^{3}$ phantom with capillary tubes $3 \mathrm{~mm}$ in length (open on the upper surface) drilled in it (Fig. 4a). A second one was designed for the determination of the transaxial spatial resolution. It consists of a cylinder with Derenzo-like geometry placed inside a holder cylinder $20 \mathrm{~mm}$ in diameter and $30 \mathrm{~mm}$ in length (Fig. 4b). The two above phantoms were used for spatial resolution determination of FOV20 and FOV40. Due to the dimensions of phantom in Fig. 4a), it can not be placed at vertical position because the cameras would crash with it during their rotation motion. For that reason it can be used only for axial spatial resolution determination. Moreover, as the spatial resolution for the FOV60 and FOV80 are worse than those for FOV20 and FOV40, we need the capillaries to be bigger (both in diameter and in center-to-center distances) than those used for the FOV20 and FOV40. For that reason we use for FOV 40 and FOV 80 the phantom in Fig. 4c). This phantom consists of a 40x40x4.5 $\mathrm{mm}^{3}$ orthoedro with Derenzo-like geometry, placed inside a 40x40x9.5 mm $\mathrm{mm}^{3}$ holder (Fig. 4c). In all cases the center-to-center distance between holes is twice the hole diameter.

Total acquisition time was $45 \mathrm{~min}$ using $50 \mathrm{MBq}$ of a ${ }^{99 \mathrm{~m}} \mathrm{Tc}$ solution. The images were reconstructed using the 3D- OSEM algorithm and 5 iterations $(5$ subsets for the singlepinhole, except for FOV20 where 15 subsets were considered, and 15 subsets for the multi-pinhole data). These parameters (number of subsets and number of iterations) were optimised for single-pinhole and multi-pinhole configurations as a balance among the image quality (both spatial resolution and noise), time required for image reconstruction, and system matrix size.

\section{III.D FUSED IMAGES. ANIMAL STUDIES}

A mouse image was recorded with the ALBIRA PET/SPECT/CT system following the procedure described in Ref. 29. The experiment was conducted with the approval of the Notre Dame Institutional Animal Care and Use Committee, (IACUC 12-115). Mouse was anesthetized by isofluorano inhalation $\left(2.5 \%\right.$ in $\left.\mathrm{O}_{2}\right)$. As part of the dosing and imaging protocol, $37 \mathrm{MBq}$ of ${ }^{99 \mathrm{~m}} \mathrm{Tc}-\mathrm{MDP}$ (Methyl Diphosphonate) was injected into a nude hairless mouse (SKH1, 6 weeks old male, 27g weight) via a catheter, and after 30 min to allow for desired biodistribution of the probe, a SPECT image was acquired. The SPECT scan was made through 60 views, FOV40 and multi-pinhole configuration, for a total acquisition time of $30 \mathrm{~min}$. The image was reconstructed using the 3D-OSEM algorithm, with a total of 5 iterations and 15 subsets, using a voxel size of $0.5 \times 0.5 \times 0.5$ $\mathrm{mm}^{3}$. CT data were acquired with the x-ray source operating at $45 \mathrm{kV}, 400 \mu \mathrm{A}$, and 600 views for a total recording time of $7 \mathrm{~min}$. CT images were reconstructed with the $\mathrm{FDK}^{24}$ algorithm. After the SPECT and CT acquisitions, 3.7 MBq of $\mathrm{Na}^{18} \mathrm{~F}$ was injected via the catheter without moving the animal. After a $30 \mathrm{~min}$ period to allow for desired 
biodistribution, a PET scan was acquired (10 min integration time). The PET images were reconstructed using the 3D-MLEM algorithm with a total of 12 iterations.

The fusion of the images recorded with PET, SPECT and CT subsystems was accomplished using a $\mathrm{C}++$ specifically developed software, which produces the spatial transformation matrix for the registration by using data derived from the geometric calibration of the system.

The trimodal imaging study resulted in a total dose to the mouse of about $13.7 \mathrm{cGy}$ (10 cGy for CT, 1.7 cGy for SPECT, and about 2 cGy for PET scan).

\section{RESULTS AND DISCUSSION}

\section{IV.A CT}

\section{IV.A.1 Spatial resolution}

The measured Modulation Transfer Function (MTF) for CT subsystem is shown in Fig. 5. Error estimation for the curve is about 5\% derived from the gaussian fitting of the single recorded image in the spatial domain. If we assume that the limiting spatial resolution corresponds to the point where the MTF drops to $10 \%$, we can infer that the spatial resolution of the CT subsystem is about 11 line pairs per $\mathrm{mm}$.

This value is compatible with the FWHM of the gold wire reconstructed image, in which a final value of $85 \mu \mathrm{m}$ was obtained for the CT spatial resolution.

\section{IV.A.2 Image uniformity and noise characteristics}

The results show a significant worsening of CT image uniformity in those configurations having better spatial resolution (Table II). These values can be explained if we consider that voxel size of the reconstructed image diminishes as the spatial resolution improves for the different configurations considered (Table I).

This decrease in voxel size worsens DEV\% and N\% to such a degree that it cannot be compensated for by an increase in exposure time that is produced when the number of views increases for the medium and high resolution configurations.

However, the rise in the number of projections (i.e. exposure time) increases the radiation dose to the animal during the scan. Nevertheless, the objective was not to get three configurations with the same noise characteristics but to have the two configurations with better noise properties, and a third one with better spatial resolution. The configurations with larger voxel sizes $(\geq 0,25 \mathrm{~mm})$, which have better noise properties, permit to identify most of the structures and organs inside a mouse or a rat. These two configurations are oriented to quantitative studies and extraction of the attenuation maps for PET and SPECT attenuation corrections. The $0,125 \mathrm{~mm}$ voxel size configuration is oriented to structures which require a slightly higher spatial resolution like bones. In this kind of studies, the large differences in attenuation values between bone and soft tissue tolerate a slight degradation in noise properties.

\section{IV.B SPECT}

\section{IV.B.1 Sensitivity}

SPECT Sensitivity variation with the energy window lead us to select an energy window of $\pm 15 \%$ for both, single- and multi-pinhole configurations (Fig. 6a), as it 
seems to be the best suited value for optimizing SPECT performance without significant contribution of scattered and background events.

A maximum sensitivity of about $700 \mathrm{cps} / \mathrm{MBq}\left(7.8 \times 10^{-2} \%\right)$ was obtained with the multi-pinhole, whereas about $200 \mathrm{cps} / \mathrm{MBq}\left(2.2 \times 10^{-2} \%\right)$ was achieved with the singlepinhole (Fig. 6b). As expected, the sensitivity deteriorates as the FOV increases.

\section{IV.B.2 Spatial resolution}

An estimation of the axial and transaxial spatial resolution for the various FOVs considered are obtained (Fig. 7) from the SPECT reconstructed images. It can be concluded (Fig. 7a) that there is a worsening of the spatial resolution when the multipinhole collimator is used (about 20\%). The spatial resolution has been estimated taking into account that the spatial resolution is better than a given Derenzo-like phantom capillary diameter size, if the profile of the peaks overlap in such a way that the counts measured in the middle point $\left(h_{3}\right)$ are lower or equal to half of the averaged detected counts in the maximum of the peaks $\left(\mathrm{h}_{1}+\mathrm{h}_{2}\right) / 4$ (Fig. $\left.7 \mathrm{~b}\right)$.

Nevertheless, the sensitivity improvements justify the use of this kind of collimator in SPECT devices. The increase in the sensitivity can be visually appreciated when comparing Figs. 7c) and d). Concerning spatial resolution performance, the best configuration corresponds to the single-pinhole collimator and FOV20, which achieves a value of about $0.8 \mathrm{~mm}$ for the spatial resolution, both in the axial and the transaxial (Fig. 7b) plane, which corresponds to a volumetric resolution of about $0.5 \mu \mathrm{l}$.

\section{IV.C PET}

The performance of the PET component of our system has been determined and extensively described by the authors in a previous work. ${ }^{23} \mathrm{We}$ only refer here the values obtained for the most significant parameters. The spatial resolution measured at the FOV center was 1.55, 1.72, and $1.67 \mathrm{~mm}$ FWHM for radial, tangential, and axial image profiles, respectively. A system scatter fraction of $7.5 \%$ (mouse-like phantom) and $13 \%$ (rat-like phantom) were obtained, while the maximum noise equivalent count rate (NECR) were $16.9 \mathrm{kcps}$ at $12.7 \mathrm{MBq}(0.37 \mathrm{MBq} / \mathrm{ml})$ for the mouse-like phantom and $12.8 \mathrm{kcps}$ at $12.4 \mathrm{MBq}(0.042 \mathrm{MBq} / \mathrm{ml})$ for the rat-like phantom The peak absolute sensitivity in the center of the FOV is $2 \%$ for $350-650 \mathrm{keV}$ energy window. As expected, when comparing SPECT and PET performance, SPECT exhibit higher spatial resolution (especially if the single-pinhole collimator is used) while PET shows higher sensitivity.

\section{IV.D FUSED IMAGES. ANIMAL STUDIES}

In Fig. 8 we show the in vivo trimodal PET/SPECT/CT image of a mouse recorded as described in III.D. No attenuation correction was made on SPECT and PET acquired data. From Fig. 8, it could be concluded that the obtained PET/SPECT/CT scanner image possesses the required features (especially spatial resolution) needed for small animal imaging. However, it should be noted that ALBIRA axial field of view (especially for PET subsystem) is limited and in some cases the entire animal can not be observed in a single acquisition. Nevertheless, the system is designed in such a manner that bed motion in the axial direction allows the "virtual" elongation of the axial FOV up to $170 \mathrm{~mm}$. This technique, based on the image fusion made by software, is limited to static studies and implies an increase in the acquisition time. 


\section{CONCLUSIONS}

We have designed a small animal PET/SPECT/CT scanner. The gamma ray subsystems (SPECT and PET) use a detector made up of monolithic scintillator crystals. SPECT's performance measurements (tomographic spatial resolution and sensitivity) for similar aperture size and radius of rotation were comparable to the results obtained from other small animal SPECT systems based on pixellated scintillator crystals. ${ }^{3,17,20}$ The energy resolution of our system $(13 \%$ at $140 \mathrm{keV})$ is worse than that of CZT semiconductor based systems $\left(5 \%{ }^{30}, 10 \%{ }^{31}\right)$ but comparable to other scintillator based systems (Table III). PET's spatial resolution, sensitivity, and scatter fraction are comparable with that obtained with much more complex crystal pixelated PET systems. ${ }^{23}$

Our PET/SPECT/CT device produces high-quality images suitable for studies with small animals. Although further improvements are foreseen in the near future for the PET/SPECT/CT scanner we have presented in this work, it is already commercially available (ALBIRA PET/SPECT/CT multimodal system, Oncovision GEM-Imaging S.A.). These improvements mainly consist of a PET equipped with 3 rings and an axial FOV of $120 \mathrm{~mm}$, together with modifications within the MA-PMT's resistive current divider network in order to increase PET counting rate capabilities. Moreover, development of a gamma camera for the SPECT subsystem equipped with a monolithic scintillator crystal larger $\left(10 \times 10 \times 0.4 \mathrm{~cm}^{3}\right)$ than that used so far $\left(5 \times 5 \times 0.4 \mathrm{~cm}^{3}\right)$ has been planned. New geometries, including more apertures, for the multi-pinhole collimators are being investigated in order to increase sensitivity without great loss of spatial resolution performance. Finally, although attenuation correction for mice and rats can be expected to be smaller than in humans, its contribution is by no means negligible. Work is on-going for use of registered CT data for SPECT and PET attenuation correction.

\section{ACKNOWLEDGMENT}

This study was supported by the Spanish Plan Nacional de Investigación Científica, Desarrollo e Innovación Tecnológica (I+D+I) under Grant No. FIS2010-21216-CO2-01 and Valencian Local Government under Grant PROMETEO 2008/114. The authors also thank Brennan Holt for checking and correcting the text. 


\section{References:}

a) Author to whom correspondence should be addressed. Electronic mail: filsan@i3m.upv.es

${ }^{1}$ D.P. McElroy, L.R. MacDonald, F.J. Beekman, Y.C. Wang, B.E. Patt, J.S. Iwanczyk, et al., "Performance evaluation of A-SPECT: a high resolution desktop pinhole SPECT system for imaging small animals," IEEE Trans Nucl Sci. 49, 2139-2147 (2002).

2 D.A. Weber, M. Ivanovic, D. Franceschi, S.E. Strand, K. Erlandsson, M. Franceschi, et al., "Pinhole SPECT: an approach to in vivo high resolution SPECT imaging in small laboratory animals," J. Nucl Med. 35, 342-348 (1994).

${ }^{3}$ F. van der Have, B. Vastenhouw, R.M. Ramakers, W. Branderhorst, J.O. Krah, C. Ji, S. G. Staelens, and F. J. Beekman, "U-SPECT-II: an ultra-high-resolution device for molecular small-animal Imaging," J. Nucl. Med. 50, 599-605 (2009).

${ }^{4}$ T. Zeniya, H. Watabe, T. Aoi, K.M. Kim, N. Teramoto, T. Takeno, et al.,"Use of a compact pixellated gamma camera for small animal pinhole SPECT Imaging," Ann. Nucl. Med. 20, 409-416 (2006).

${ }^{5}$ N. Kubo, S. Zhao, Y. Fujiki, A. Kinda, N. Motomura, C. Katoh, et al., "Evaluating performance of a pixel array semiconductor SPECT system for small animal Imaging," Ann. Nucl Med. 19, 633-639 (2005).

${ }^{6}$ E. Lage, J.J. Vaquero, A. Sisniega, S. España, G. Tapias, M. Abella, A. Rodrí1guezRuano, J.E. Ortuño, A. Udías, and M. Desco, "Design and performance evaluation of a coplanar multimodality scanner for rodent imaging," Phys. Med. Biol. 54, 5427-5441 (2009).

${ }^{7}$ S.A. Kis, I. Lajtos, M. Emri, L. Tron, G. Opposits, T. Bükki, Gy. Hegyesi, J. Imrek, I. Valastyán, J. Molnár, D. Novák, and L. Balkay, "Performance test of the MiniPET-II small animal scanner according to the NEMA NU-4 standard," IEEE Nuclear Science Symposium Conference Record , 3185-3189 (2009).

${ }^{8}$ Q. Bao, D. Newport2, M. Chen, D.B. Stout, and A.F. Chatziioannou, "Performance Evaluation of the Inveon Dedicated PET Preclinical Tomograph Based on the NEMA NU-4 Standards," J. Nucl. Med. 50, 401-408 (2009).

${ }^{9}$ R. Prasad, O. Ratib, and H. Zaidi, ,'Performance Evaluation of the FLEX Triumph XPET Scanner using the National Electrical Manufacturers Association NU-4 Standards," J. Nucl. Med. 51, 1608-1615 (2010).

${ }^{10}$ M. C. Huisman, S. Reder, A. W. Weber, S. I. Ziegler, and M. Schwaiger, "Performance evaluation of the Philips MOSAIC small animal PET scanner," Eur. J. Nucl. Med. Mol. Imaging 34, 532-540 (2007).

${ }^{11}$ N. Belcari, A. Del Guerra, A. Bartoli, D. Bianchi, M. Lazzarotti, L. Sensi, L. Menichetti, M. Lecchi, P.A. Erba, G. Mariani, G.U. Corsini, and P. Sgadó, "Evaluation of the performance of the YAP-(S)PET scanner and its application in neuroscience," Nucl. Instrum. Methods A 571, 18-21 (2007).

${ }^{12}$ H. Zhang, Q. Bao, N.T. Vu, R.W. Silverman, R. Taschereau, B.N. Berry-Pusey, A. Douraghy, F.R. Rannou, D.B. Stout, and A.F. Chatziioannou, "Performance Evaluation of PETbox: A Low Cost Bench Top Preclinical PET Scanner," Mol. Imaging Biol. 13, 949-961(2011).

${ }^{13}$ J.S. Kim, J.S. Lee, K.Ch. Im, S.J. Kim, S.Y. Kim, D.S. Lee, and D.H. Moon, "Performance Measurement of the microPET Focus 120 Scanner," J. Nucl. Med. 48, 1527-1535 (2007). 
${ }^{14}$ M. Cañadas, M. Embid, E. Lage, M. Desco, J.J. Vaquero, and J.M. Pérez, "NEMA NU 4-2008 Performance Measurements of Two Commercial Small-Animal PET Scanners: ClearPET and rPET-1,” IEEE Trans. Nucl. Sci., 58, 58-65, (2011).

${ }^{15}$ R. Prasad, O. Ratib, and H. Zaidi, "NEMA NU-04-based performance characteristics of the LabPET-8 ${ }^{\mathrm{TM}}$ small animal PET scanner," Phys. Med. Biol. 56, 6649-6664 (2011). ${ }^{16}$ S.R. Cherry, "Multimodality Imaging: Beyond PET/CT and SPECT/CT,"Semin. Nucl. Med. 39, 348-353(2009).

${ }^{17}$ A. del Guerra and N. Belcari, "State-of-the-art of PET, SPECT and CT for small animal Imaging," Nucl. Instrum. Methods A 583, 119-124 (2007).

18 A.B. Hwang, B.L. Franc, G.T.Gullberg, and B.H. Hasegawa, "Assessment of the sources of error affecting the quantitative accuracy of SPECT imaging in small animals," Phys. Med. Biol.53, 2233-2252 (2008).

19 A.B. Hwang and B.H. Hasegawa, "Attenuation correction for small animal SPECT imaging using X-ray CT data," Med. Phys. 32, 2799-2804 (2005).

${ }^{20}$ K. Magota, N. Kubo, Y. Kuge, K. Nishijima, S. Zhao, and N. Tamaking, "Performance characterization of the Inveon preclinical small-animal PET/SPECT/CT system for multimodality Imaging”, Eur. J. Nucl. Med. Mol. Imaging 38, (2011)742752.

${ }^{21}$ R.J. Smith, J.S. Karp, and G. Muehlllehner, "The count rate performance of the volume imaging PENN-PET scanner,” IEEE Trans. Med. Imag. 13, 610-618 (1994).

${ }^{22}$ F. Sánchez, M. M. Fernández, M. Giménez, J. M. Benlloch, M. J. Rodríguez-Alvarez, F. García de Quirós, Ch. W. Lerche, N. Pavón, J. A. Palazón, J. Martínez, and A. Sebastiá, "Performance tests of two portable mini gamma cameras for medical applications," Med. Phys. 33, 4210-4221 (2006).

${ }^{23}$ F. Sánchez, L. Moliner, C. Correcher, A. González, A. Orero, M. Carles, A. Soriano, M. J. Rodríguez-Alvarez, L. A. Medina, F. Mora, and J. M. Benlloch, "Small animal PET scanner based on monolithic LYSO crystals: Performance evaluation," Medical Physics 39, 643-653 (2012).

${ }^{24}$ N. L. Feldkamp, L.C. Davis, and J.W. Kress, ’Practical cone-beam algorithm”, J. Opt. Soc. Am. A, 1 612-619 (1984).

${ }^{25}$ D. Bequé, J. Nuyts, G. Bormans, P. Suetens, and P. Dupont, "Characterization of Pinhole SPECT Acquisition Geometry," IEEE Trans. Med. Imag. 22, 599-612(2003).

${ }^{26}$ D. Bequé, J. Nuyts, P. Suetens, and G. Bormans,"Optimization of Geometrical Calibration in Pinhole SPECT," IEEE Trans. Med. Imag. 24, 180-190 (2005).

${ }^{27}$ Y. Wang and B. Tsui, "Pinhole SPECT With Different Data Acquisition Geometries: Usefulness of Unified Projection Operators in Homogeneous Coordinates," IEEE Trans. Med. Imag. 26, 298-308 (2007).

${ }^{28}$ R. J. Jaszczak, K. L. Greer, C.E. Jr Floyd, C. C. Harris, and R. E. Coleman, "Improved SPECT quantification using compensation for scattered photons," J. Nucl. Med. 25, 893-900(1984).

${ }^{29}$ S. E.Chapman, J. M. Diener, T. A. Sasser, C. Correcher, A. J. González, T.V. Avermaete, and W. M. Leevy, "Dual tracer imaging of SPECT and PET probes in living mice using a sequential protocol," Am. J. Nucl. Med. Mol. Imaging 2, 405414(2012).

${ }^{30}$ D. Wagenaar, et al., "A multi-ring small animal CZT system for simultaneous SPECT/MRI imaging," J. Nucl. Med. 48 (Supplement 2), 89P (2007).

${ }^{31}$ H. Kim, L. R. Furenlid, M. J. Crawford, D. W. Wilson, H. B. Barber, T. E. Peterson, W. C. Hunter, Z. Liu, J. M. Woolfenden, and H. H. Barrett, "SemiSPECT: a smallanimal single-photon emission computed tomography (SPECT) imager based on eight cadmium zinc telluride (CZT) detector arrays," Med. Phys. 33, 465-474 (2006). 



\section{Figure Caption:}

Fig. 1. Schematic view of the entire PET/SPECT/CT system, showing its main geometric parameters.

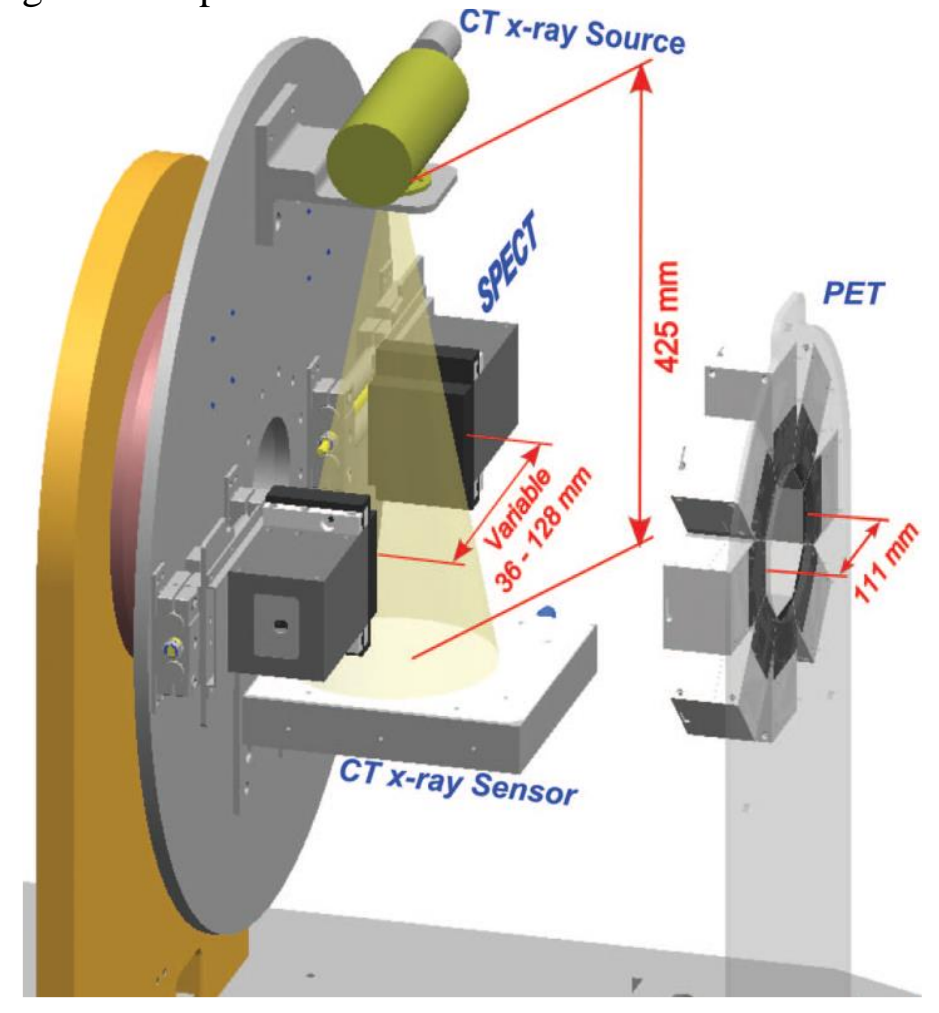

Fig. 2. (a) Schematic view of the single pinhole collimator with a knife-edge type aperture. (b) Schematic view of multi-pinhole configuration, with tilted apertures. $b_{1}$ refers to the minimum distance such that the center of the FOV can be seen by all the apertures. $b_{2}$ refers to the radius of rotation (FOV20, FOV40, FOV60, and FOV80 $b_{2}=20,32,47$, and $66 \mathrm{~mm}$, respectively).

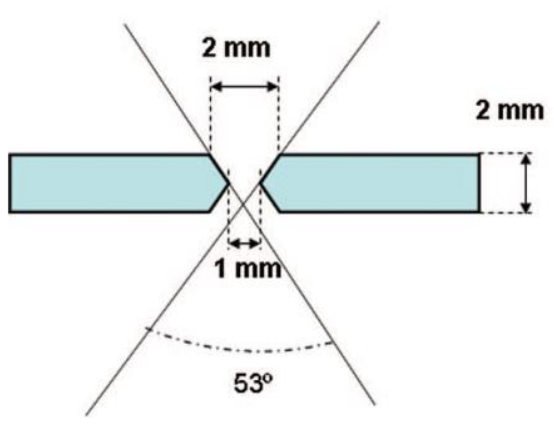

(a)

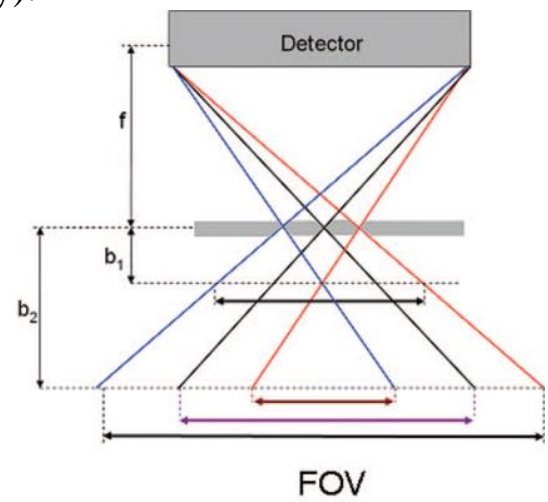

(b) 
Fig. 3. The small animal whole system PET/SPECT/CT.

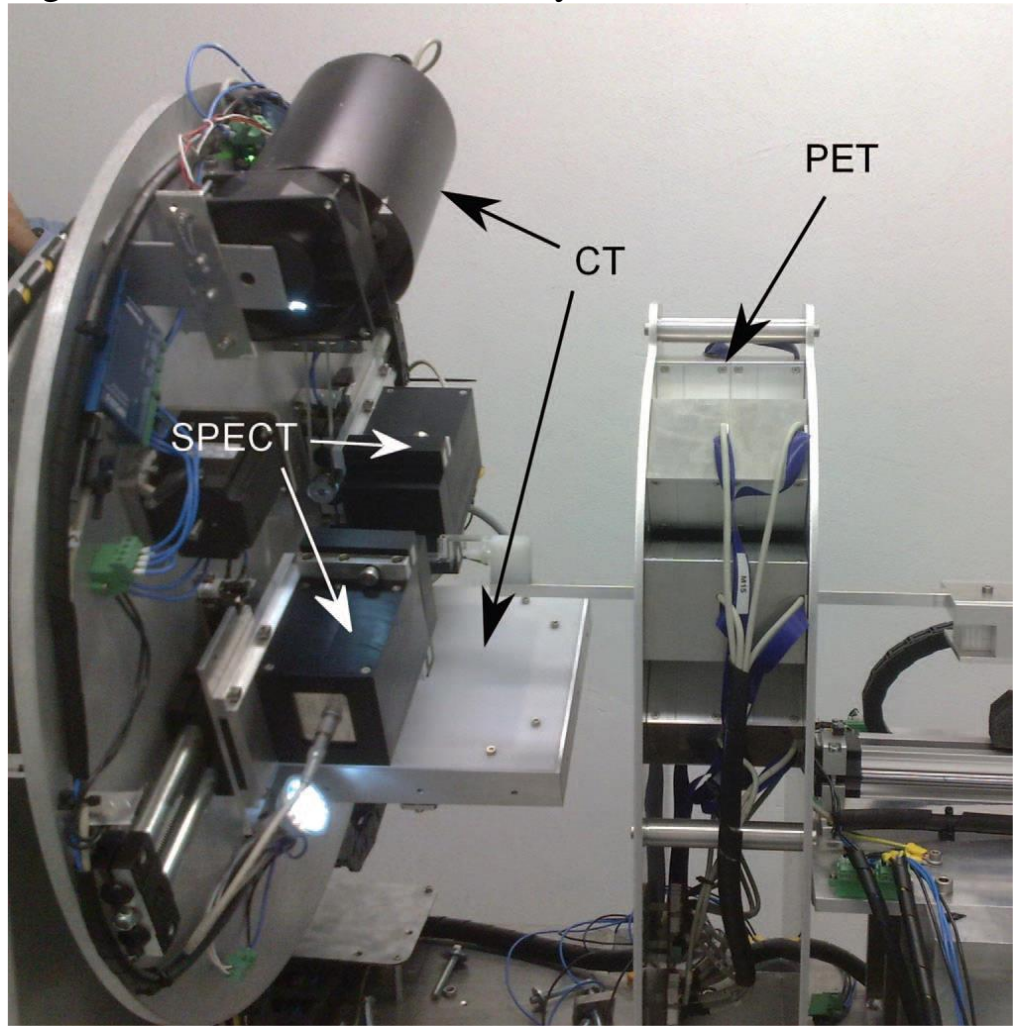

Fig. 4. Derenzo-like phantoms used for SPECT spatial resolution determination. (a) Phantom used for axial resolution for FOV20 and FOV40. (b) Phantom used for transaxial resolution for FOV20 and FOV40. (c) Phantom used for FOV60 and FOV80. Numbers in the images refer to the diameter in $\mathrm{mm}$ of the drilled tubes.

(a)

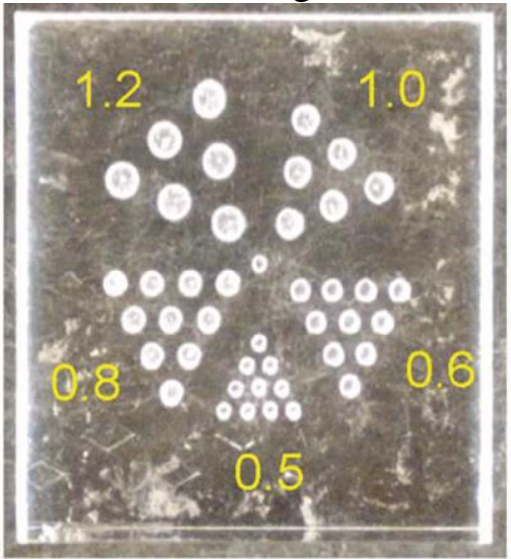

(b)
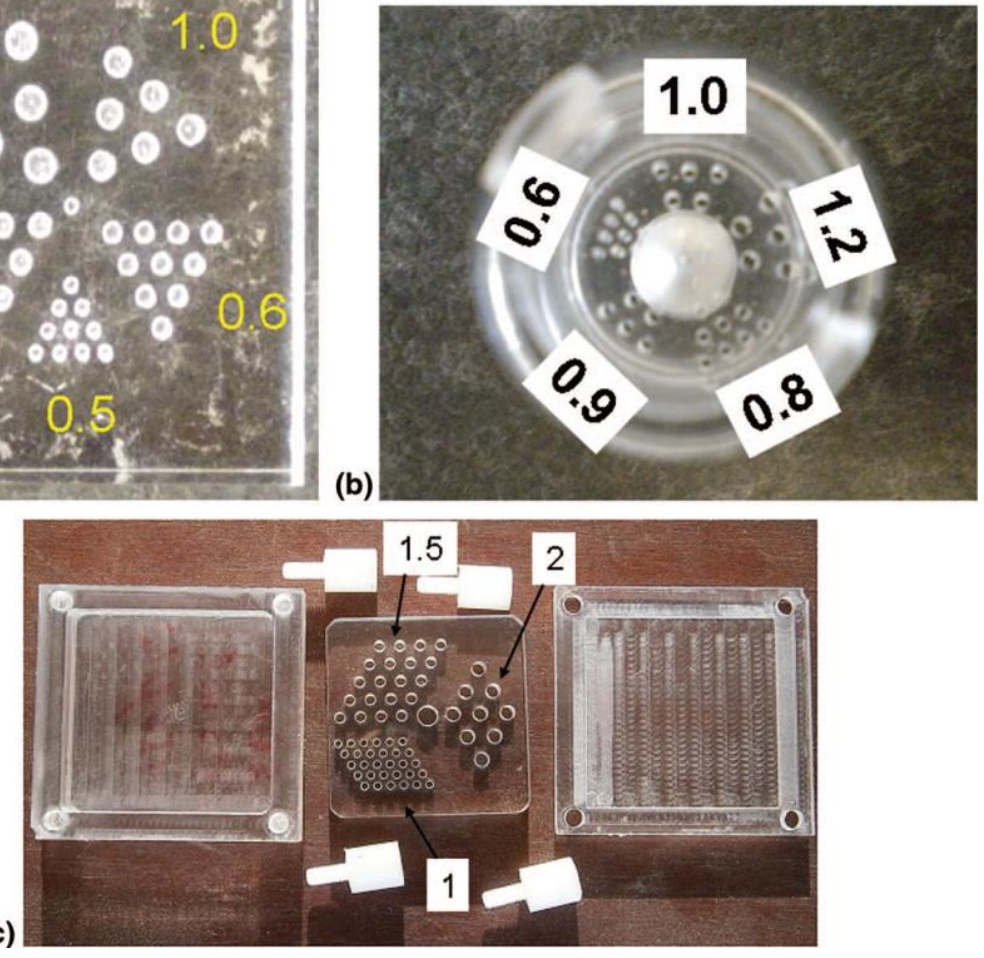
Fig. 5. Measured MTF curve of the CT subsystem using a gold wire $50 \mu \mathrm{m}$ in diameter placed in the center of the FOV.

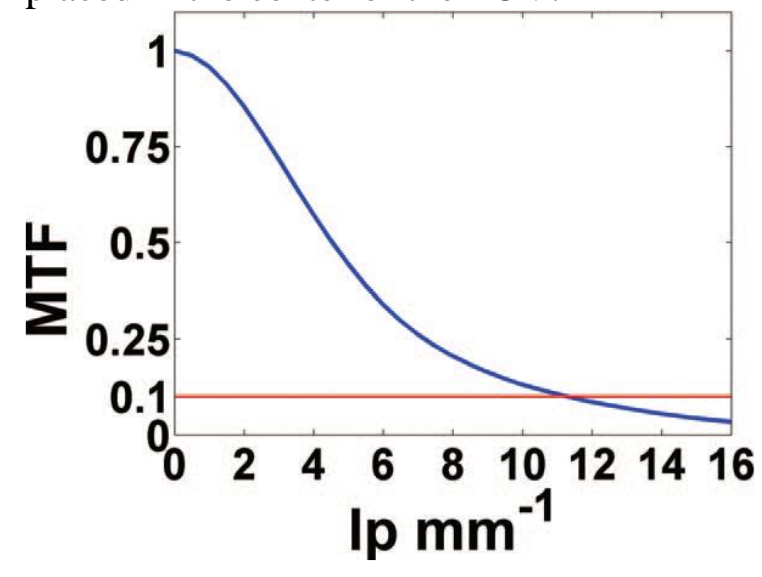

Fig. 6. a)Variation of SPECT sensitivity with the energy window for the single- and multi-pinhole collimator at the FOV20 configuration. b) SPECT sensitivity variation with the FOV for single- and multi-pinhole collimator configuration. The energy window is set to $\pm 15 \%$.
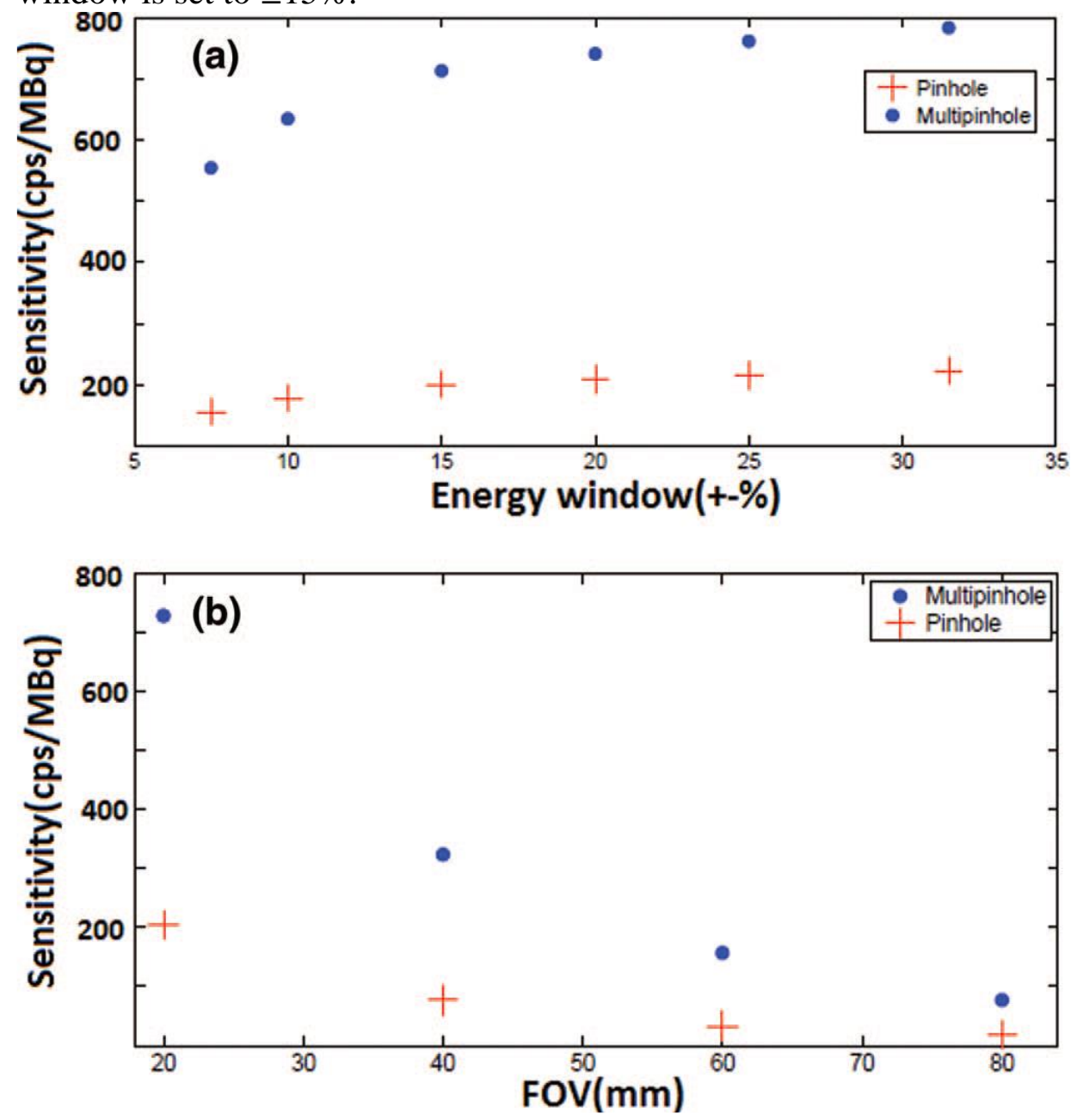
Fig. 7. a) Transaxial spatial resolution for the SPECT subsystem for the single- and multi-pinhole configuration at different FOVs. Although not showed, no significant differences are observed for the axial component. b) Transaxial profile of the $0.8 \mathrm{~mm}$ diameter line sources obtained with the transaxial resolution phantom (Fig. 4b) using the single-pinhole collimator and FOV20 configuration. See the text for $h_{1}, h_{2}$, and $h_{3}$ meaning. c) reconstructed image in the transaxial plane of the phantom of Fig. 4 b) using the single-pinhole and FOV20 configuration, voxel size $0.25 \times 0.25 \times 0.25 \mathrm{~mm}^{3} . \mathrm{d}$ ) same as c) but using the multi-pinhole with voxel size of $0.35 \times 0.35 \times 0.35 \mathrm{~mm}^{3}$. In c) and d), the central slice is shown.
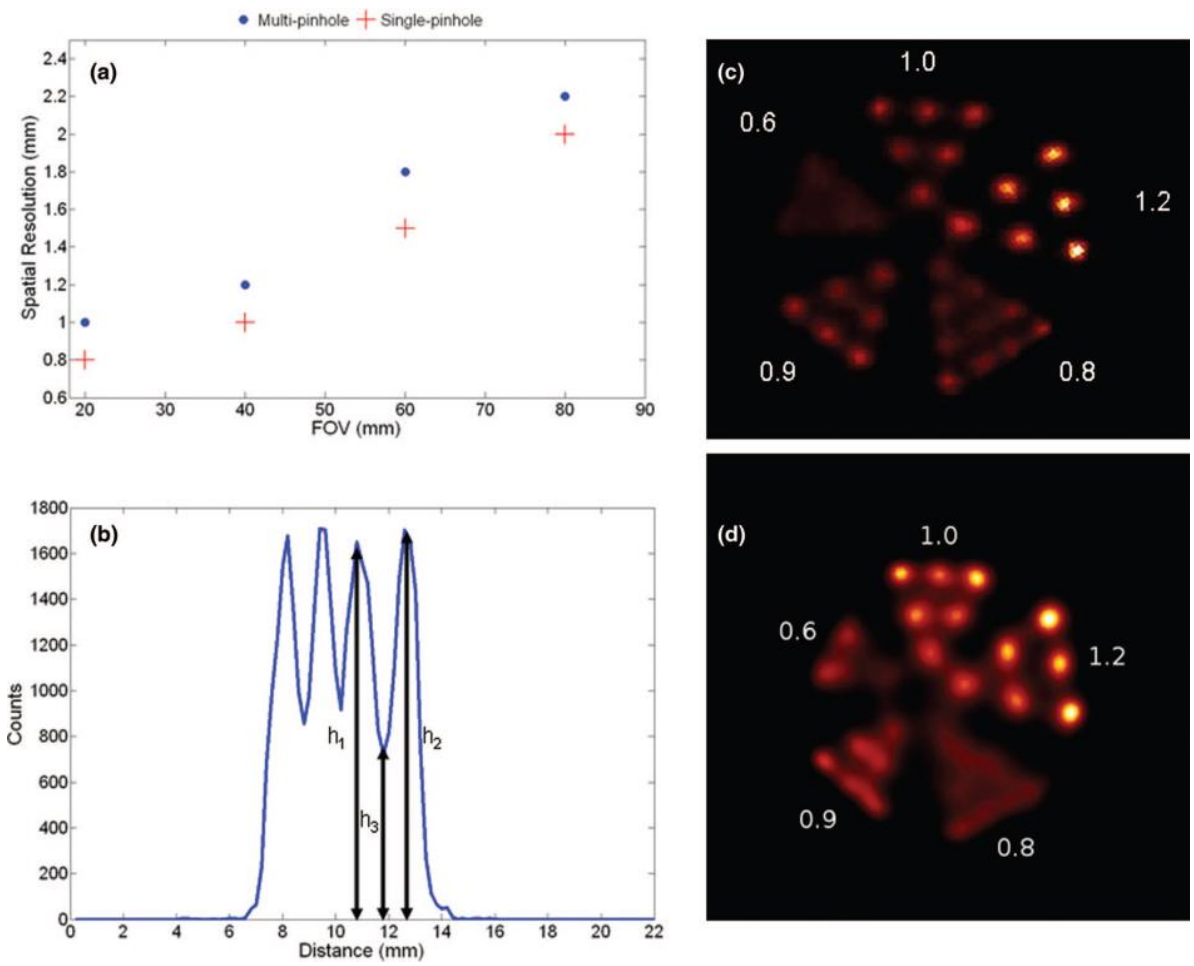

Fig. 8. Trimodal in vivo imaging of a mouse obtained by using ${ }^{99 \mathrm{~m}} \mathrm{Tc}-\mathrm{MDP}$ (SPECT) and $\mathrm{Na}^{18} \mathrm{~F}$ (PET) as tracers. Up (from left to right): CT, PET, and fused PET/CT. Down (from left to right): SPECT, fused SPECT/CT, and fused PET/SPECT/CT image.

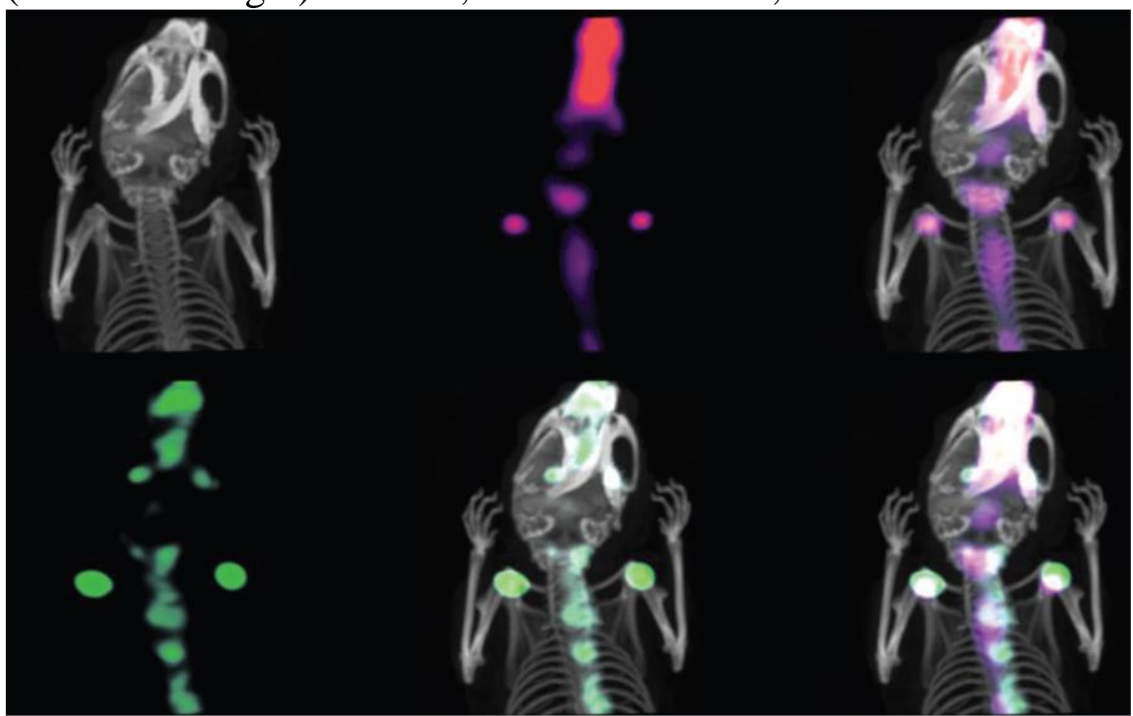




\section{TABLES}

Table I. Albira CT working configurations.

\begin{tabular}{|c|c|c|c|}
\hline Configuration & Views & Voxel size $\left(\mu \mathrm{m}^{3}\right)$ & $\begin{array}{c}\text { Detector } \\
\text { binning }\left(\mu \mathrm{m}^{2}\right)\end{array}$ \\
\hline Highest resolution & 1000 & Variable $^{\mathrm{a}}$ & $50 \times 50$ \\
\hline High resolution & 600 & $125 \times 125 \times 125$ & $100 \times 100$ \\
\hline Medium resolution & 400 & $250 \times 250 \times 250$ & $100 \times 100$ \\
\hline Low resolution & 250 & $500 \times 500 \times 500$ & $100 \times 100$ \\
\hline
\end{tabular}

a From $35 \times 35 \times 35 \mu^{3}$ up to $125 \times 125 \times 125 \mu \mathrm{m}^{3}$. For determination of the spatial resolution this configuration with a voxel size of $8 \times 8 \times 8 \mu \mathrm{m}^{3}$ was used .

Table II. DEV(\%) and N(\%) obtained with the CT for different configurations.

\begin{tabular}{|c|l|l|}
\hline Configuration & $\mathrm{DEV}(\%)$ & $\mathrm{N}(\%)$ \\
\hline High resolution & 11.5 & 3.5 \\
\hline Medium resolution & 5.5 & 1.67 \\
\hline Low resolution & 2.7 & 0.86 \\
\hline
\end{tabular}


Table III. ALBIRA PET/SPECT/CT system performance compared with other trimodal systems commercially available.

\section{PET}

ALBIRA Inveon $^{20} \quad$ Triumph $^{\mathrm{a}} \quad$ Vector $^{\mathrm{b}}$

Number of crystals Dimensions (mm)

$8(40 \times 40 \times 10) 25600(1.4 \times 1.4 \times 10) \quad 6144(2 \times 2 \times 12 / 14) 3$ clinical detectors $(595 \times 472)$

Spatial Resolution(mm)

Energy Resolution

$\begin{array}{ll}1,55 & 1,4 \\ 14 \% & 18 \% \\ \text { Yes } & \text { No } \\ 2 \% & 6.7 \%\end{array}$

1,35

$<1$

DOI

Sensitivity

$2 \%$

$6.7 \%$

N/A

$>12 \%(\mathrm{NaI}(\mathrm{Tl}))$

Yes (phoswich) No

$6 \%$

$<12000 \mathrm{cps} / \mathrm{Mbq}$

\section{SPECT}

Detector Heads

2

Spatial Resolution(mm) 0,8

up to 4

1

Energy Resolution $\quad 15 \%$

$14 \%$

Sensitivity(cps/Mbq) 700

$>1000$

30-300

up to 4 (CZT) 3

0,4

0,25

Energy Range (keV) 50-250

$4,5 \%$

$>12 \%(\mathrm{NaI}(\mathrm{Tl}))$

6500

12000

25-250

20-511

\section{CT}

Spatial Resolution $(\mu \mathrm{m}) 85$

15

50

$>80$

Max. kV

50

80

80

65

Max. mA

1

Focal Spot Size $(\mu \mathrm{m}) \quad 35$

$1 \quad 0,5$

0,65

variable 6-60

N/A

N/A

${ }^{a}$ http://www.gammamedica.com/pre_triumph_trimodality_system.html

${ }^{\mathrm{b}} \mathrm{http} / / / \mathrm{www}$.milabs.com/imaging-solutions/vector-simultaneous-spectpet/ 